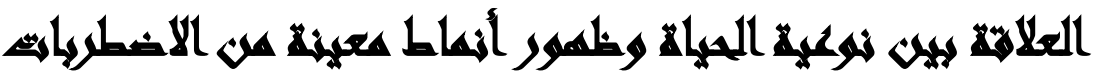

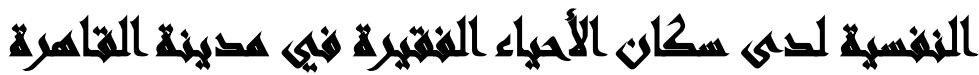

[9]

أحمد مصطفى العتيق(')- محمود عبد الحميد حسين(ץ) - سمية إسماعيل صادق العدوي

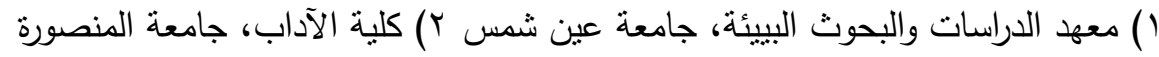

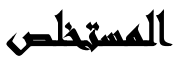

تتهـ الدول النامية ظاهرة التضخم الحضرى ونموه المتزايد بصورة مستمرة وزيادة الكثافة السكانية و اقتراب عدد السكان في كثير من البيئات الحضرية الى حد النتبع النئ (سمير إبراهيم

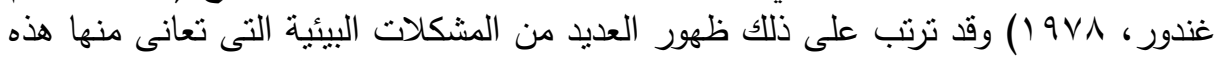

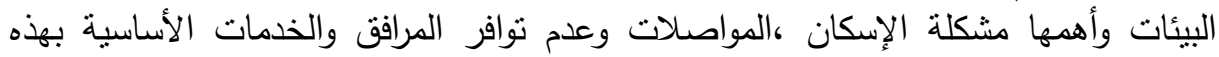
المدن (على عبد الرازق حطب،

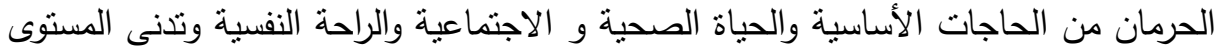

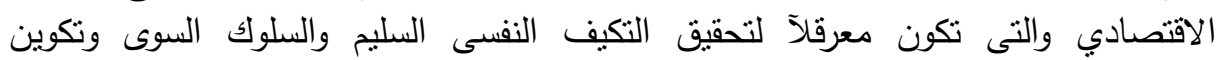

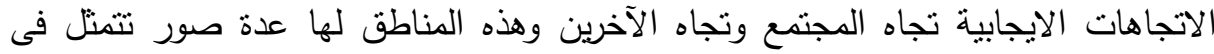

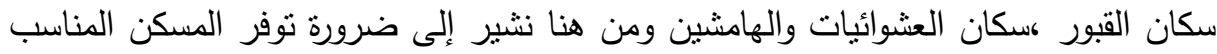

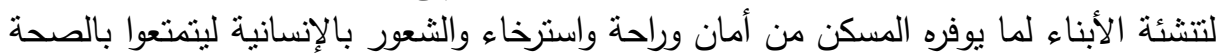

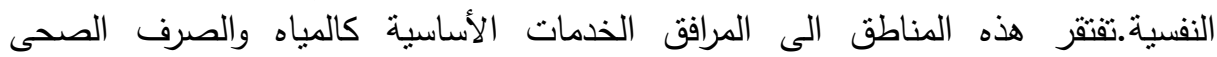

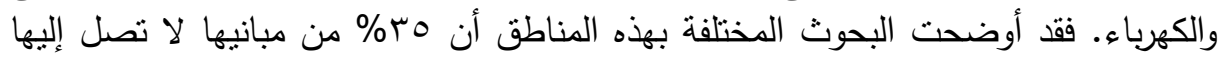

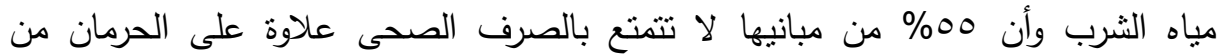

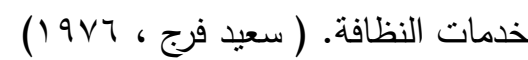
هدف البحث: وتهدف الدراسة الراهنة للتعرف الته على أحدى المشكلات الهامة التى يعانى منها

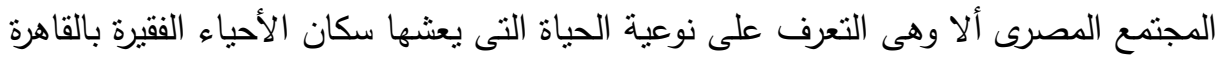

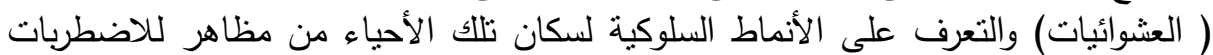
النفسية.

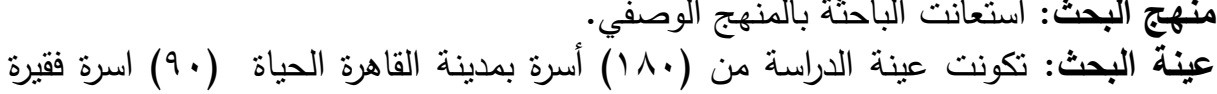
و (9) أسرة اخري متوسطة. أدوات البحث:استخدمت الباحثون عدة عدة مقاييس في هذا البحث لتحقيق الأهداف المرجوة من البحث وهي ما يلي: 


$$
\text { ا (مقياس الاضطربات النفسية (اكتئاب-قلق) إعداد الباحثة }
$$

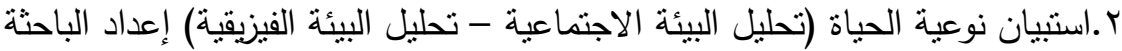

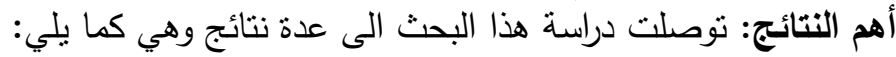

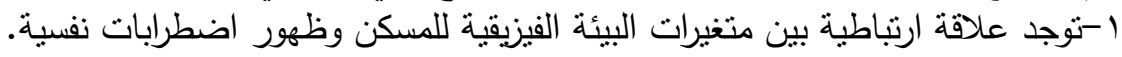

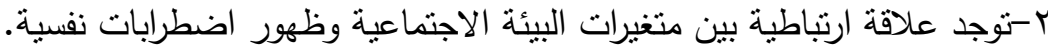

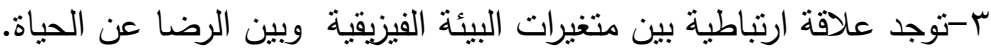

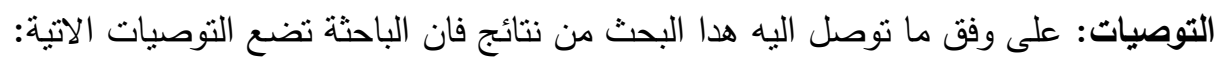

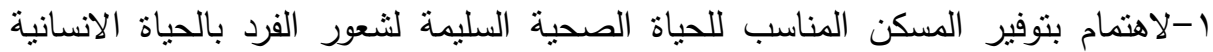

الكريمة حيث ينوفر في المسكن الخدمات والمرافق اللازمة للابتعاد عن عشوائية السكن.

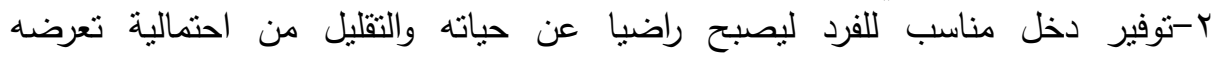
للاضطربات النفسية.

\section{ranatl}

إن الإنسان خلق لكي يحيى ويعيش في سعادة، ولابد من مقومات لهذه الحياة، حياة

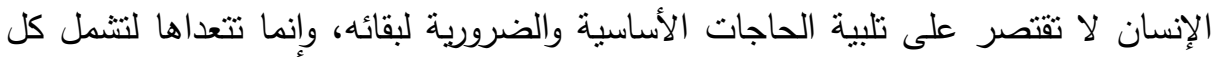
ما يحسن نوعية الحياة للإنسان وامتبازه عن باقي المخلوقات، كالمقومات العقلية والنفسية والوجدانية والاجتماعية مع ما تتضمنه من قدرة على التفكير وعلاقات اجتماعية ومعتقدات دينية وقيم ثقافية وحضارية وأوضاع مالية واقتصادية، تمكن كل شخص أن يحدد ما الثئ

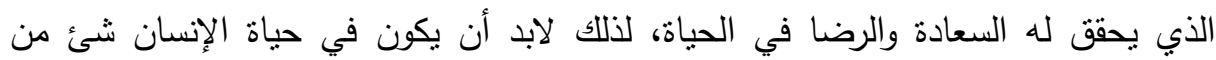
النوعية والجودة لتحقيق ذللك.( محمد نصحى غريب، 919 (1) نتشه الدول النامية ظاهرة التضخم الحضرى ونموه المتزايد بصورة مستمرة وزيادة الكثافة السكانية و اقتراب عدد السكان

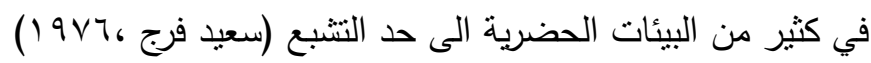
وقد نرتب على ذلك ظهور العديد من المشكلات البيئية التى تعانى منها هذه البيئات

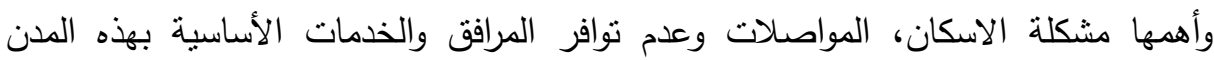

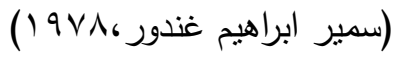
فهناك مناطق فى مدينة القاهرة تتمثل فيها بيئات الحرمان من الحاجات الأساسية والحياة الصحية و الاجتماعية والراحة النفسية وتدنى المستوى الاقتصادى والتى تكون معرقلاً لتحقيق

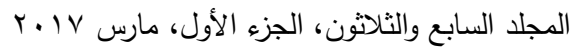


التكيف النفسى السليم والسلوك السوى وتكوين الاتجاهات الايجابية تجاه المجتمع وتجاه الاخرين (عبد الرحمن الطريري، (99 (19)

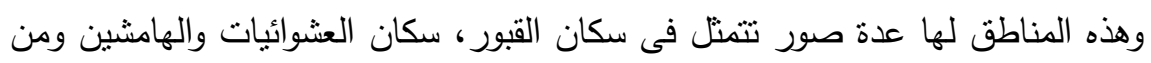
هنا نشير الى ضرورة توفر المسكن المناسب لنتشئة الابناء لما يوفره المسكن من أمان وراحة لهندان واسترخاء والثعور بالإنسانية ليتمتعوا بالصحة النفسية).

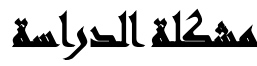

تعنبر ظاهرة السكن العشوائى من أكثر المشاكل البيئية التى تهدد المدن فى العالم بشكل عام وفى العالم الثالث بثكل خاص .حيث تؤدى المجتمعات العشوائية الى تشويه وتدهور حالة البيئة السكنية وتؤثر سلبآ على المناطق المحيطة بها لما تعانيه من تدهور فى نوعية

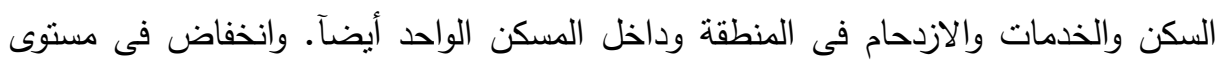

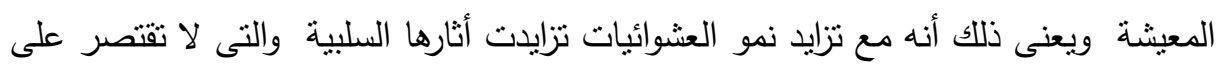

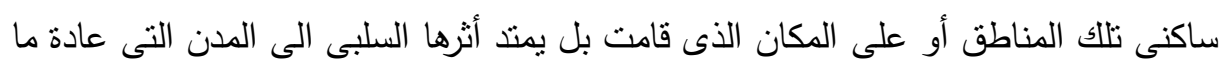
تقوم هذه المناطق على اطرفها. ونتيجة اقامتها بدون تخطيط وبدون مرافق وخدمات أصبحت تشكل عبئً على الخدمات فى المدينة كالنقل، التعليم، الصحة، الصرف الصنئ الصحى ومياه الثرب والكهرباء.

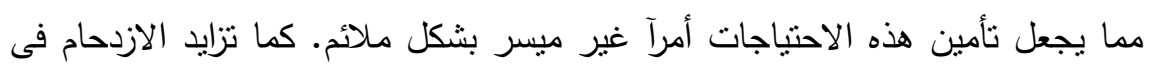

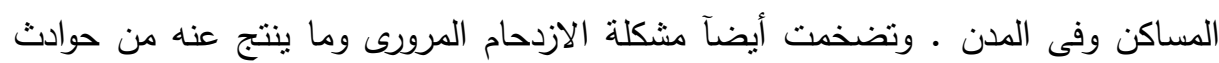

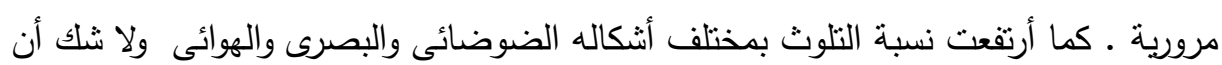
لهذا أثاره التى تتعكس على ظروف حياة الأفراد وأدأهم لوظائفهم وقدراتهم ومستقبل الاجيال

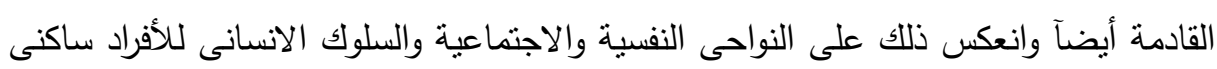

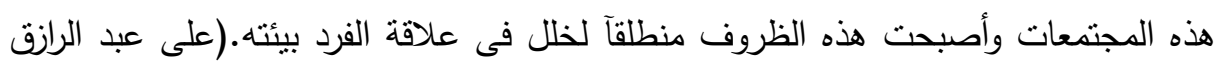




\section{أهساهيم التراسة}

يمكن تحديد أهداف الدراسة فى النقاط التالية: ا.التعرف على العلاقة بين نوعية الحياة وظهور بعض الاضطربات النفسية لدى سكان الاحباء الفقيرة.

r.الكثف عن العلاقة بين متغيرات البيئة الفيزيقية والضغوط النفسية والاجتماعية . r.الكثف عن علاقة بين متغيرات البيئة الأجنماعية وظهور أضطربات نفسية.

\section{هغوضر القراسمة}

ا.توجد علاقة أرتباطية ذات دلالة إحصائية بين متغيرات البيئة الفيزيقية للمسكن وظهور أضطربات نفسية. r.توجد علاقة أرتباطية ذات دلالة احصائية بين متغيرات البيئة الأجتماعية وظهور أضطربات نفسية. r.نوجد علاقة أرتباطية ذات دلالة احصائية بين متغيرات البيئة الفيزيقية وبين الرضا عن الحباة.

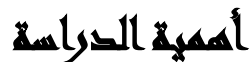

جاءت أهمية تلك الدراسة للتعرف على المشكلات الاساسية لسكان الاحياء الفقيرة بالقاهرة التي تمنلها المساكن العشوائية نظرآ للاهتمام بنوعية الحياة في هذه المناطق وما يوجد بها من مشكلات عديدة ولكن لكل منطقة مشكلاتها الخاصة والتى تختلف من منطقة لاخرى تبعا لاختلاف الظروف البيئية. إذن تكمن أهمية هذه الدراسة فى التعرف على نوعية الحياة

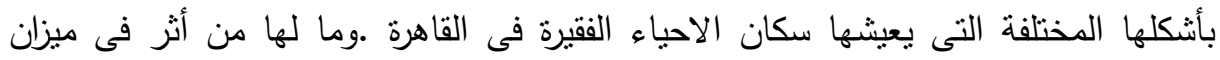
الصحة النفسية والاجتماعية للاقراد وما يصاحبها من ظهور بعض الاضطربات النفسية كالعنف، العدوان ،التعصب، الاغتراب، قلق، أكتئاب، لامبالاة، انعزال، احساس بالوحدة. هذه المظاهر تعتبر مدخلات للافات المرضية التى تحدث في هذه الاونة مثل انتشار المخدرات

$$
\text { المجلد السابع والثلاثون، الجزء الأول، مارس V V. T }
$$


وانتشار التطرف الدينى والسياسى وظهور الرهاب الاسود وجميع صور العنف. وايضا ترجع اهمية هذه الدراسة لأهمية المناخ البيئى الذى يعيش فيه" الانسان " واستخدمت لفظ انسان

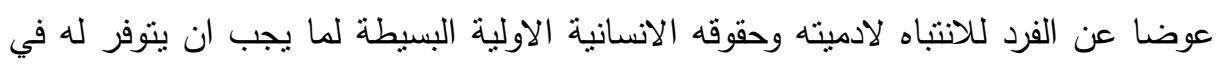

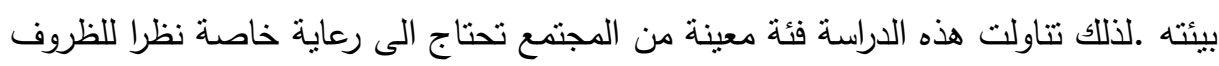

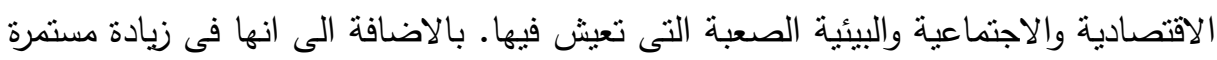
في العقود الاخيرة، وينعكس ذلك على نوعية حياة الافراد وعلى علاقتهم ببيئتهم وعلى صحتهم النفسية والجسدية واحساسهم بالرضا عن الحياة.

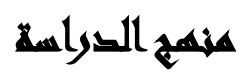

استعانت الباحثة بالمنهج الوصفي من حيث اهتمامه بالخصائص الفيزيقية لبيئة معينة في تأثثرها على السلوك الاجتماعي وتأثرها بذلك اي انه يهنم بدراسة العلاقة بين الانسان والبيئة.

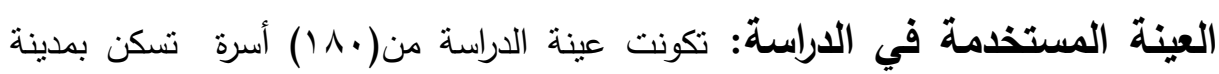
القاهرة والتعرف على نوعية الحياة الاجتماعية والفيزيقية الخاصة بهم واثر ذلك او ارتباطه بظهور بعض الاضطرابات النفسية. ولتحقيق ذلك طبقت الدراسة على (·وع) اسرة فقيرة و ( • (9) اسرة اخري منوسطة.

\section{السراسايت الماريها}

هناك دراسات اهتمت بالقياس المباشر لنوعية الحياة من خلا دراسة بعض الأبعاد

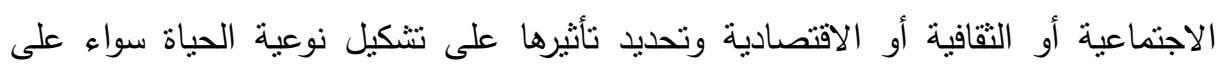
مستوى المجتمع القومي أم المجتمع المحلي أم على مستوى الفرد والأسرة: ا - دراسة كاتلين وكريستوفر (Kattlen and Kristopher,2002): "عن دور وقت الفراغ في قياس نوعية الحياة"

وكان هدفها الأساسي هو محاولة اختيار وقياس دور وقت الفراغ في الكثف عن نوعياة الحياة للفرد، وذلك بتحديد الأهمية النسبية لكل من المؤشرات الخاصة بالموقع (المكان)

$$
\text { المجلد السابع والثلاثون، الجزء الأول، مارس V V. }
$$


كالتسهيلات المتاحة للاستمتاع بوقت الفراغ ومصادر وقت الفراغ وكذللك تلك المؤشرات الخاصة بالفرد ذاته (كالرضا عن تجاربه الخاصة بوقت الفراغ) ودور كل منها في التتبؤ بنوعية الحياة.

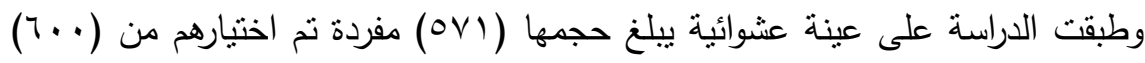
أسرة بأستراليا، وكثفت الاراسة عن النتائج الآتية: - أن إسهامات وقت الفراغ المتمركزة على الفرد هي الأكثر أهمية في التتبؤ بنوعية الحياة،

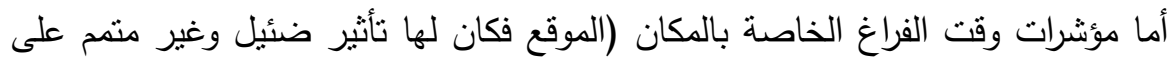

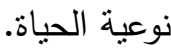
- أن الذين يندمجون في الأنشطة الاجتماعية بصورة أكبر والذين يكون لهم شعور أكثر رضاء عن المزايا المستمدة من وقت الفراغ كالراحة والاستجمام والترفيه عن النفس ومقابلة الأصدقاء.. وغيرها، هم الذين يستمتعون بنوعية حياة أفضل. - تلعب المتغيرات الديموجرافية دوراً في إدراك الناس وخبرتهم بنوعية حياتهم (السن، النوع،

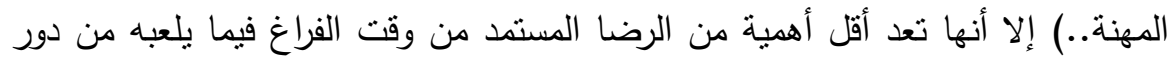
في التنبؤ بنوعية الحياة. r - دراسة مارجريت بولما (Margret M. Polma, 1990): كانت تهدف إلى إلقاء الضوء على علاقة الأبعاد الدينية بالرضا عن جوانب الحياة ككل وقد أجريت على عينة قوامها

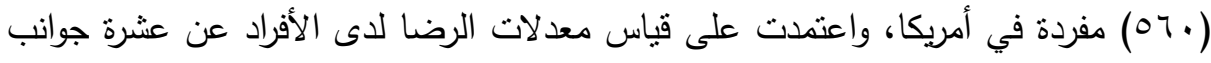

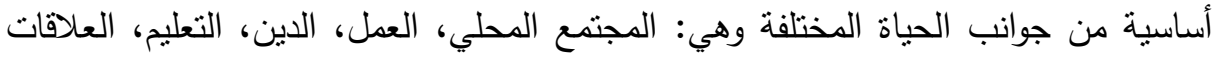
الاجتماعية، الأسرة، الزواج، مستوى المعيشة، الصحة، العمل المنزلي.

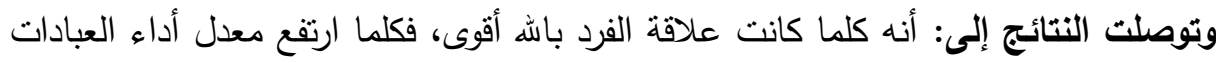
كلما أدى ذلك إلى تقليل درجات الخوف والتوتز والقلق وزيادة معدلات القناعة والراحة النفسية

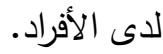




\section{James R. Peacock and ب ب براسة مارجريت بولما وجيمز بيكو} (Margaret. M. Polma,1999)

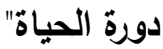

واهتمت بالدور الذي يلعبه التدين المفرد (زيادة التدين) في التأثير على الرضا عن الحياة

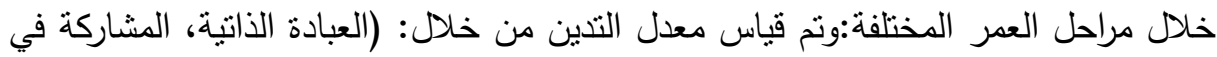
الطقوس والثُعائر الدينية العامة، التفاعل الديني، تفضيل التدين العام أو الخاص). وتم قياس هند

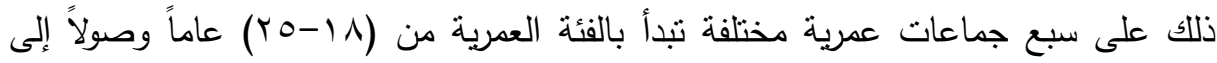

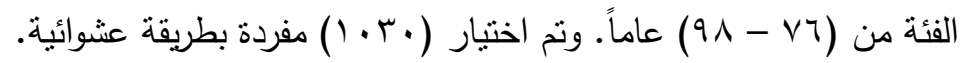
وتوصلت النتائج إلى: - وجود علاقة إيجابية مهمة بين العمر وبين مقياس التدين.

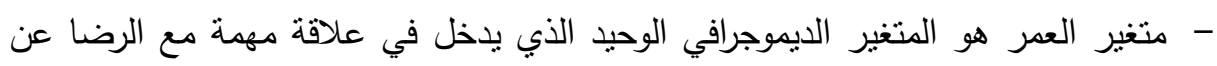

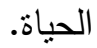
- أن التدين المفرط هو الأفضل في التتبؤ بالرضا عن الحياة طوال فترات العمر . ع - دراسة ميشيل هيجرتي حول "الرضا عن الحياة"، 999 19 1:

واعتمد على نظريتين من النظريات الكبرى الخاصة بدراسة نوعية الحياة وهي: نظرية القدرة على الحياة والتي تفترض أن الأفراد يقومون بالحكم على الرضا عن حياتهم معتمدين

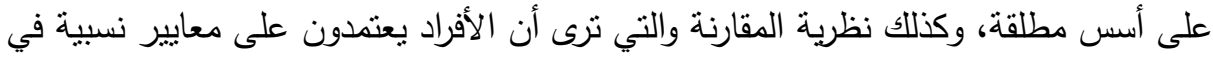

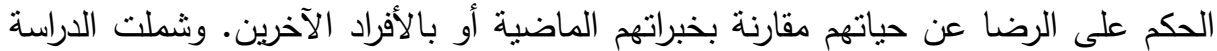
ثماني دول، وحاولت الدراسة قياس الرضا عن الحياة في ضوء التغيرات المستمرة عبر الوقت

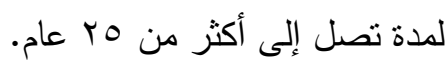
وتوصلت النتائج إلى أنها اتفقت مع العديد من الدراسات السابقة في أن المستوى المطلق لنصيب الفرد من الدخل القومي كان له أكبر نأثثير على الرضا عن الحياة، بينما كثفت

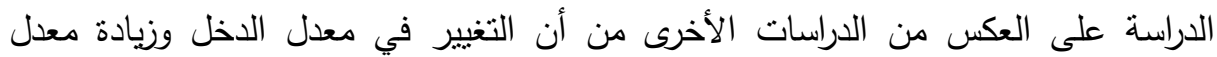
الاستهلاك كان له تأثثر أكبر على تشكيل معدل رضا الفرد عن حياته.

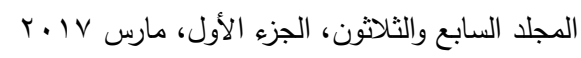




\section{ه - دراسة جوزيف سيرجي وكورنيل تيري(Joseph Cornwell Terri,2001:}

تهذف إلى الكثف عن نأثثر الرضا عن المجتمع المحلي وما به من خدمات على تحقق

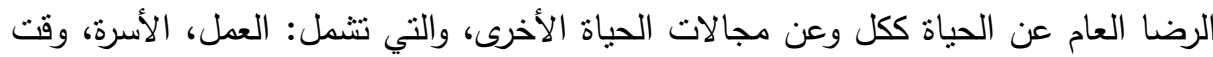
الفراغ، وطبقت الدراسة على عينة متتوعة من المجتمعات المحلية واعتمدت على نظرية الإفاضة التي تفترض أن الرضا عن الحياة برتبط وظيفياً بالرضا عن كل مجالات الحئه الحياة الأخرى ومجالاتها الفرعية (كالرضا عن المجتمع المحلي، الأسرة، الزواج، العمل، الحياة

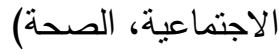

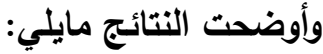

1-الثعور بالرضا أو عدم الرضا عن المجتمع المحلي ينتكل من خلال الثعور بالرضا عن

$$
\text { الخدمات الحكومية، الأعمال التجارية والخدمات الأخرى. }
$$

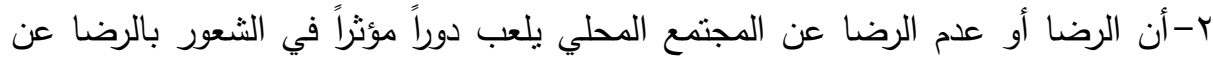

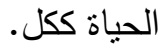

ץ-أن الرضا الثامل عن خدمات المجتمع المحلي تتكامل مع الرضا عن مجالات المجتمع

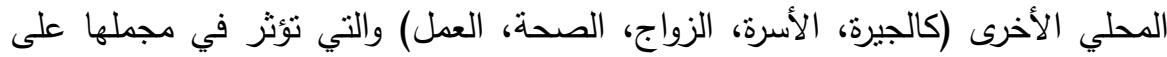
الثعور بالرضا عن الحياة ككل.

צ- دراسة جوزيف سرجي وآخرين (Joseph Sirgy, cit, al,2001)عنوان "مقياس جديد لنوعية حياة العمل" بالاعتماد على كل من نظرية الحاجة إلى الرضا

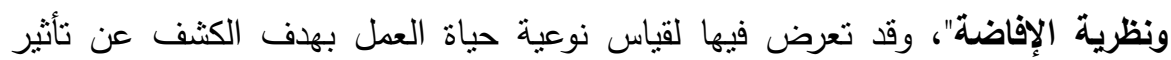
تحقيق الرضا والإثباع في مجال العمل على تحقيق الرضا في مجالات الحياة الأخرى، ومن ثم الشعور بالرضا عن الحياة ككل. وبلغت حجم العينة ( •9 ) مفردة. وقد أسفرت الاراسة عن نتيجة مهمة وهي: أن تحقيق الرضا وإثباع الحاجات المختلفة في مجال العمل تتعكس بدورها على تحقيق الرضا عن مجالات الحياة الأخرى التي تتمنل في لهي (الأسرة- الزواج- الأصدقاء- الجيرة- البيئة- الأنشطة الاجتماعية) وبالتالي تتعكس بشكل واضح على الثعور بالرضا عن الحياة ككل.

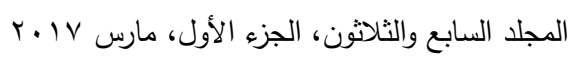




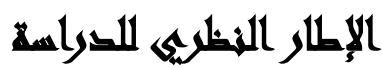

وقد شمل مفهوم نوعية الحياة عدة تعريفات نذكر منها: كانت هناك محاولات عديدة لوضع

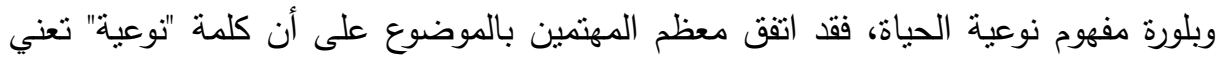

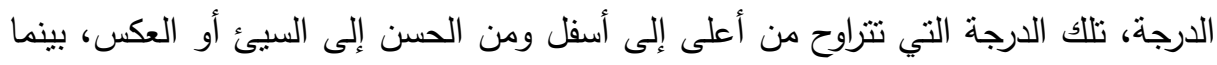

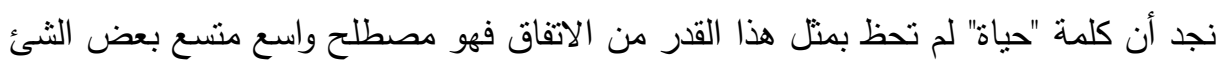
حيث يشير عند البعض إلى تلك العمليات الحيوية التي تهدف إلى بقاء الإنسان حياً (على قيد التهاف

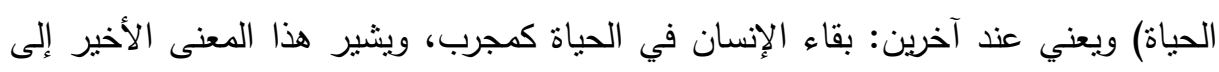
السلوك الجمعي أو حجم الرفاهية في المجتمع.

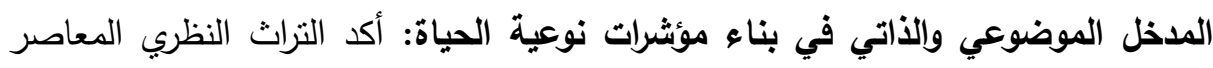

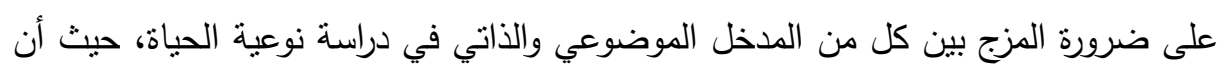

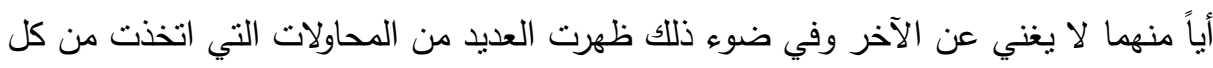

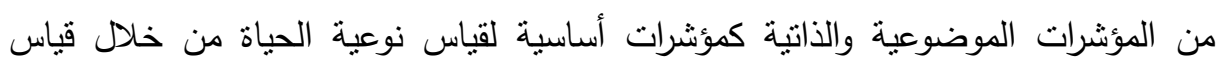

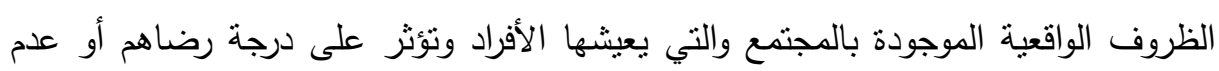

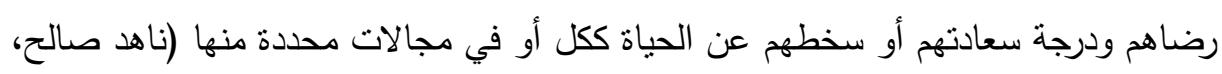

كما ظهر حديثاً الدعوة إلى ضرورة النظر إلى نوعية الحياة ككل متكامل نظرياً ومنهجياً،

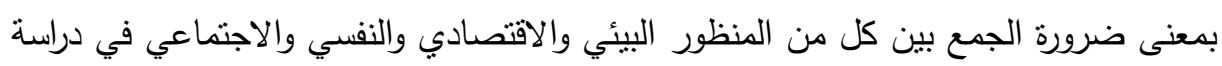
نوعية الحياة، من أجل الكثف عن الواقع الاجتماعي والظروف المجتمعية التي يعيشها

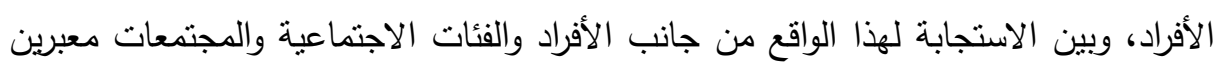
عنها بمدى الرضا أو السخط وبمدى السعادة أو التعاسة. النظريات المفسرة لنوعية الحياة:

نظرية المعنى في الحياة: تأثر فرانكل في بادئ الأمر بالفكر الفروبدي عند تفسيره للسلوك

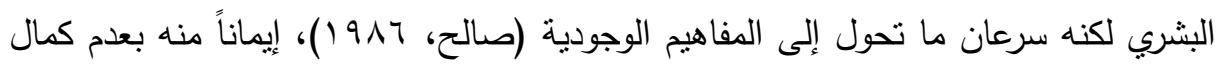
التحليل النفسي، فالإنسان من وجهة نظره أكثر من مجرد (جهاز نفسي) محكوم بغرائزه

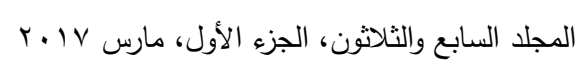


الثهوية المكبوتة كما يصوره أدرلر (Statck, 1992). ففي كثير من الأحيان لا يستجيب الإنسان لنزواته الغريزية مع تمكنه منها وإنما يستجيب إنها

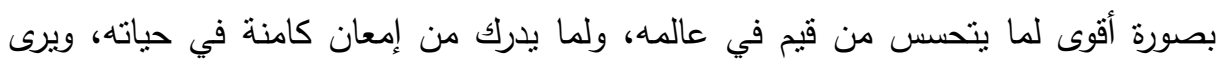

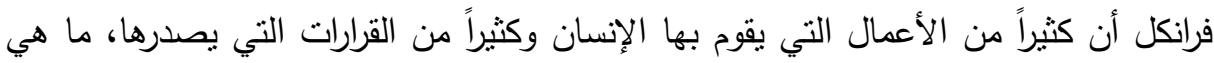
في الواقع إلا تعبيراً حقيقياً عن عملية البحث عن القيم والمعاني. ولقد أكد فرانكل أهمية القيم والمعاني في حياة الإنسان وعدها البعد الروحي الحر المسئول والمهم في تكوين شخصيته، فهو يرى بأن الكثير من الناس يستجييون ويتصرفون ويسلكون وفقاً لهذا البعد، وما عملية البحث عن المعنى والعدالة والحرية والمسؤولية والحقيقة إلا تعبيراً حقيقي عن أهية هذا لهنية البعد.

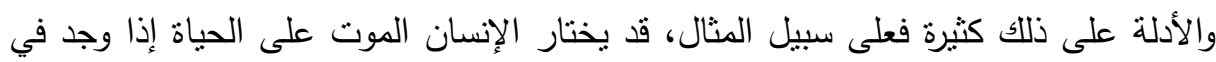

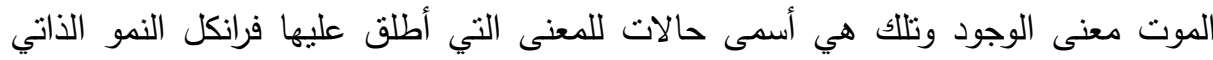

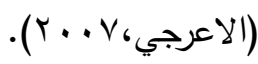

لقد صور فرانكل الإنسان على أنه وحدة لها أبعاد متفاعلة ومتكاملة هي البعد السوماتي لاعي

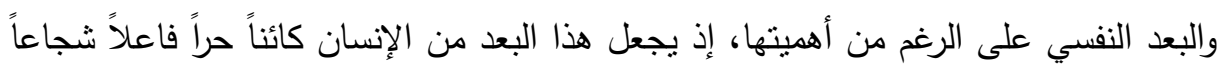

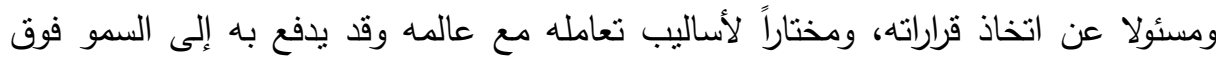
حاجاته وغرائزه النفسية والجسدية (Starck,1992).

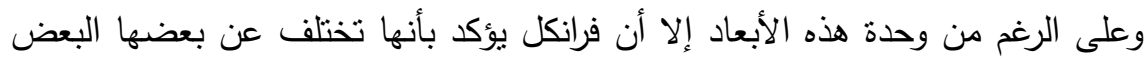
في الطبيعة والانتظام والوظيفة، وهو أمر لا يقلل من شأن كل واحد منها، فالبعد السوماتي الأي يمثل الحياة البشرية في الواقع فالبعد السوماتي يمثل الحياة البشرية في الواقع الجسدي جميعها

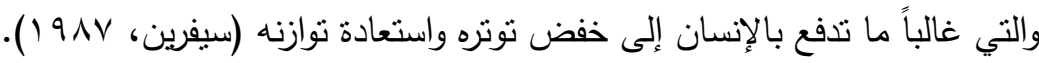
أما البعد الروحي أو (الثخص الوجودي) فيمثل لتأكل ما يدفع بالإنسان إلى زيادة توترنه

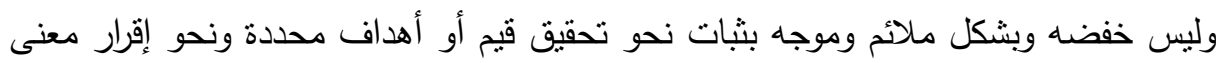

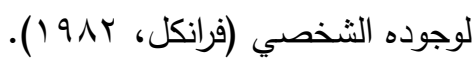


أحمد مصطفى العثيق وآخرون

\section{إجبراءاهي السراسة}

أجريت الدراسة على أسر تسكن منطقة الكيلو ه، ع عزية الهجانة بمحافظة القاهرة. الأدوات المستخدمة في الدراسة: ا (بقياس الاضطربات النفسية (اكتئاب-قلق)

r.اسنبيان نوعية الحياة (تحليل البيئة الاجتماعية - تحليل البيئة الفيزيقية) 1-مقياس الاضطرابات النفسية: (اعداد الباحثة) نم اعداد وتصميم هذا المقياس حتى

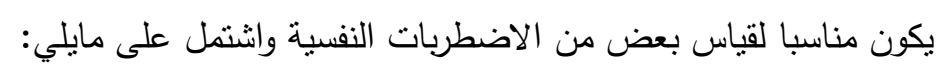

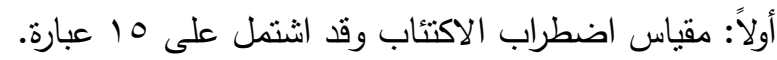
ثانيا: مقياس اضطراب القلق وقد اشتنل على 10 عبارة. واعتمدت الباحثة في صدق المقياس على:

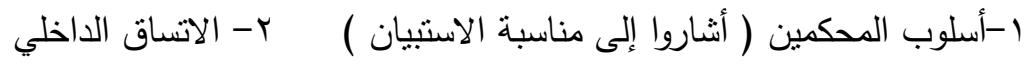

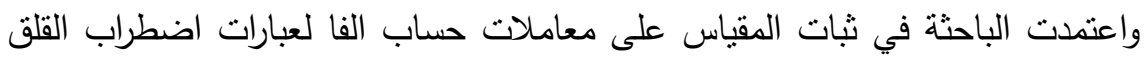
(דr, • • ) و مقياس اضطراب الاكتئاب (

r-استبيان نوعية الحياة: (اعداد الباحثة): تم اعداد وتصميم هذا المقياس حتى يكون مناسبا لقياس عدة جوانب في حياة الفرد من الناحية الاجتماعية والاقتصادية والتعليمية والصحية وقد اشتمل على مايلي: أولاً: الحالة الاجتماعية والاقتصادية للاسرة وشملت ما ولئي:

$$
\begin{aligned}
& \text { 1-مستوى التعليم للاب والام } \\
& \text { r- المهنة والوظيفة للاب والام } \\
& \text { ب-دخل الاسرة } \\
& \text { ع-نوعية تعليم الابناء } \\
& \text { ه- الانفاق الشهري للاسرة } \\
& \text { 1-نوعية الحياة الصحية والتعليمية } \\
& \text { V نوعية الحياة البيئية للمسكن }
\end{aligned}
$$


ثانيا: :نوعية الحياة الفيزيقية للمسكن

ثالثا: نوعية الحياة الاجتماعية

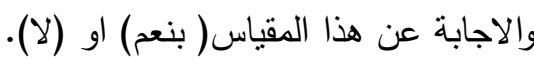

واعتمدت الباحثة في صدق المقياس على:

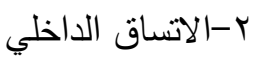

1-اسلوب المحكمين (أثناروا إلى مناسبة الاستبيان )

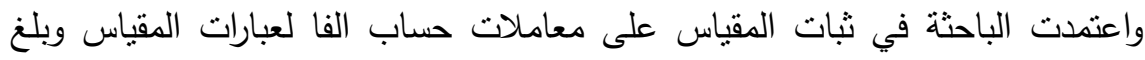

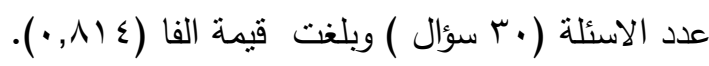

التحليل الإحصائي للمقايس: وقد تم تفريغ البيانات عن طريق البرنامج الإحصائي المعروف

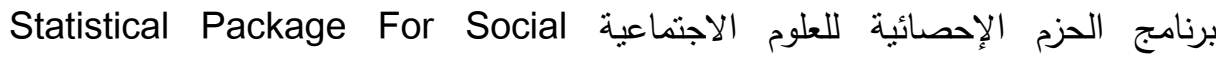
Sciences الإحصائية SPS V. 20 وتعد هذه الخطوة - تفريخ البيانات- خطوة تمهيدية لتبويب

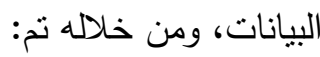
ا- اختبار الثبات من خلال معامل ألفا كرونباخ Alpha Cronbachs لاختبار ثبات

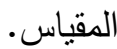

r- اختبار صدق الإتساق الداخلي من خلال معامل إرتباط بيرسون بين الأبعاد وإجمالي

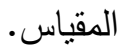
r- الإحصاءات الوصفية للبيانات من خلال جدولة البيانات في صورة جداول نكرارية (التكرار والنسبة المئوية) لأسئلة استمارات المقياس. ـ - اختبار "ت" T-Test لتوضيح الفروق بين عينة الدراسة تبعاً لمتغير النوع. 0ـ إيجاد العلاقات الإرتباطية بمعامل ارتباط بيرسون لإثبات صحة فروض الدراسة.

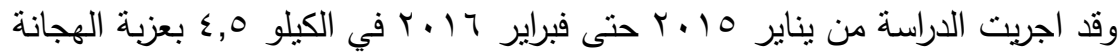
على اسر هذه المنطقة. 


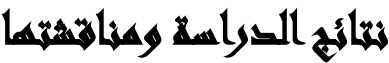

في ضوء الفروض التي طرحتها الدراسة الحالية سوف يتم عرض الننائج الخاصة بكل فرض: الفرض الأول: توجد علاقة ارتباطية بين متغيرات البيئة الفيزيقية للمسكن وظهور الفياتية

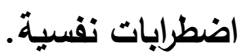
جدول(1): العلاقة الارتباطية بين مقياس نوعية الحياة الفيزيقية للمسكن وظهور اضطرابات نفسية

\begin{tabular}{|c|c|c|c|c|}
\hline \multicolumn{3}{|c|}{ مقياس نوعية الحياة الفيزيقية للمسكن } & & \multirow[b]{2}{*}{ 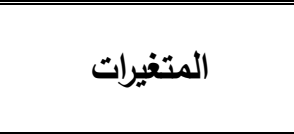 } \\
\hline إجمالي العينة & متوبياءة & أحياء فقيرة & & \\
\hline$($ (*) & $\cdot, 197-$ & $\left({ }^{* * *}\right) \cdot, 7 \leq V$ & معامل الارتباط & \multirow{2}{*}{ مقياس إضطراب } \\
\hline$\cdot, \cdots 1$ & $\cdot, \cdot V$ & $\cdot, \cdots 1$ & الالالة المعنوية & \\
\hline$($ (")"., 01 . & $\cdot, 119$ & $($ ("). , , VOV & معامل الارتباط & \multirow{2}{*}{ مقياس اضطراب القلق } \\
\hline$\cdot, \cdots 1$ & $\cdot, \cdot \Lambda$ & $\cdot, \cdots 1$ & الالالة المعنوية & \\
\hline
\end{tabular}

من الجدول السابق للعلاقة الارتباطية بين متغيرات البيئة الفيزيقية للمسكن وظهور اضطرابات نفسية يتبين الآتي: ه توجد علاقة إرتباطية ذات دلالة معنوية عند مستوى معنوية (0., •) بين مقياس نوعية

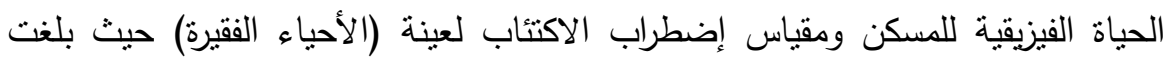

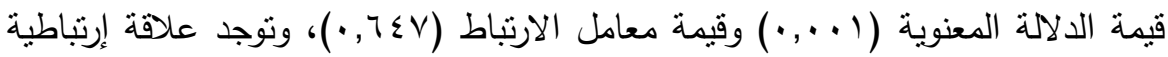

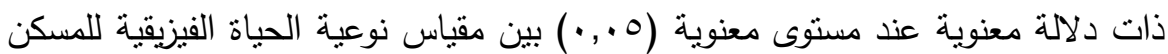
ومقياس إضطراب الاكتئاب لإجمالي العينة حيث بلغت قيمة الدلالة المعنوية (1 (.,. •) وقيمة معامل الارتباط (10, (·) )، بينما لا توجد علاقة إرتباطية ذات دلالة معنوية عند مستوى معنوية (0., •) بين مقياس نوعية الحياة الفيزيقية للمسكن ومقياس إضطراب

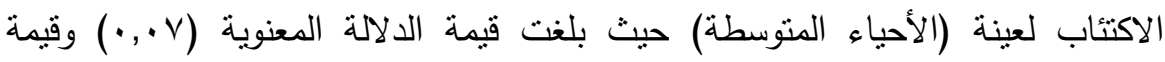
معامل الارتباط (-97 19. •). 
ك توجد علاقة إرتباطية ذات دلالة معنوية عند مستوى معنوية (0., •) بين مقياس نوعية الحياة الفيزيقية للمسكن ومقياس إضطراب القلق لعينة (الأحياء الفقيرة) حيث بلغت قيمة دئه

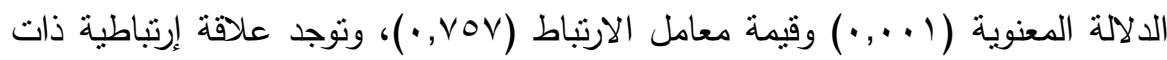
دلالة معنوية عند مستوى معنوية (0.,.) بين مقياس نوعية الحياة الفيزيقية للمسكن

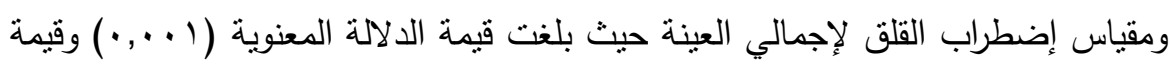
معامل الارتباط (• (0, •)، بينما لا توجد علاقة إرتباطية ذات دلالة معنوية عند مستوى لإنه معنوية (0. . •) بين مقياس نوعية الحياة الفيزيقية للمسكن ومقياس إضطراب القلق لعينة (الأحياء المتوسطة) حيث بلغت قيمة الدلالة المعنوية (1.,..•) وقيمة معامل الارتباط

ويتضح مما عرض ان نوعية الحياة الفيزيقية للمسكن لها اثر نفسي على الفرد مما قد يصيبه باضطرابات نفسية مثل القلق والاكتئاب وهدا ما اثنارت اليه الدراسة الحالية في سياقها النظري حيث ان كل من الضغوط التي يتعرض لها الفرد اي نوعية الحياة التي يعيشها الفرد تؤثر عليه سلبا او موجبا في حالته النفسية وقد اتضح دلك في الدراسة الحالية بناء على العينة

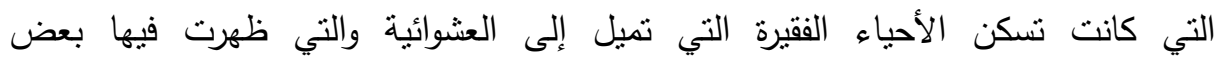
الاضطرابات النفسية كالقلق والاكتئاب بنسبة أعلى من وجودها عند العندي العينة الاخرى التي

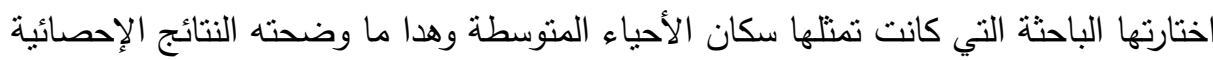
التي عرضتها الباحثة. واتضح ان نوعية ومواصفات المسكن والحي الدي يعيش فيه الفرد يؤثز

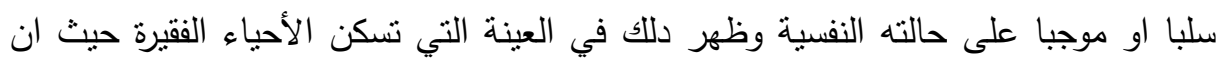
هناك نوع من الحرمان من المواصفات اللازمة للمسكن الصحي فكانت تتميز هده المساكن بالعشوائية اي ليس لديها المرافق الصحية السليمة وتفتقد التهوية الصحية للمسكن غير ان

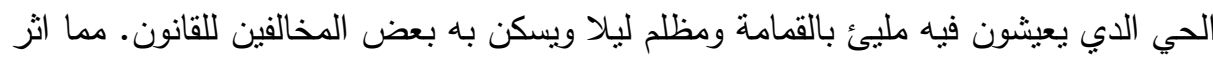

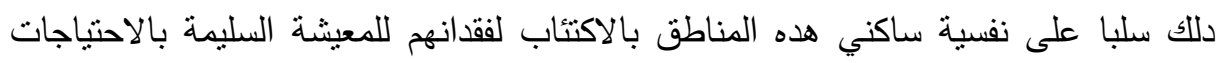

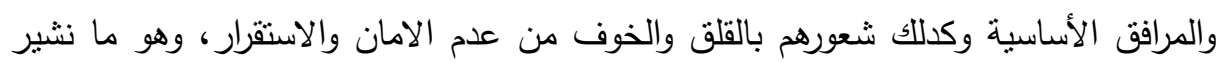

$$
\text { اليه بعدم الرضا عن الحياة. }
$$

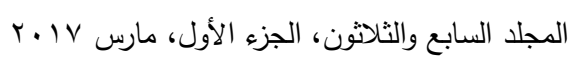


وتتفق هده النتيجة الي بعض من الدراسات السابقة التي عرضنها الباحثة: دراسة جوزيف سيرجي وكورنيل تيري ا ـ . ץ: حيث أوضحت النتائج ان الثعور بالرضا او عدم الرضا عن المجتمع المحلي ينتكل من خلال الثعور بالرضا عن الخدمات

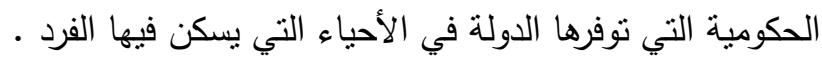
دراسة مارجريت بولما ـ99 19: حيث نوصلت نتائج هده الدراسة الى ان كلما كانت علاقة الفرد باله اقوى كلما قلل دللك من درجات الخوف والتوتر والقلق وزيادة معدلات القناعة بالحياة التي يعيشها الفرد والراحة النفسية والرضا عن مستوى المعيشة. الفرض الثاني: توجد علاقة ارتباطية بين متغيرات البيئة الاجتماعية وظهور اضطرابات

جدول(ץ): العلاقة الارتباطية بين البيئة الاجتماعية وظهور اضطرابات نفسية

\begin{tabular}{|c|c|c|c|c|}
\hline \multicolumn{3}{|c|}{ مقياس نوعية الحياة الاجتماعية } & & \multirow{2}{*}{ المتغيرات } \\
\hline إجمالّى العينة & أحياء متوسطة & أحياء فقيرة & & \\
\hline$($ ("i").,,$\leqslant \vee 7$ &., 17. & $\left({ }^{* * *}\right) \cdot, 791$ & معامل الارتباط & \multirow{2}{*}{ مقياس إضطراب الاكتئاب } \\
\hline$\cdot, \cdots 1$ & $\cdot, 1$ & $\cdot, \ldots 1$ & الالالة المعنوية & \\
\hline$\left({ }^{* * *}\right) \cdot, 0 \cdot \mathrm{V}$ & $(*) \cdot, Y Y Y$ & $\left({ }^{* * *}\right) \cdot, \vee Y q$ & معامل الارتباط & \multirow{2}{*}{ مقياس اضطراب القلق } \\
\hline$\cdot, \cdots 1$ & $\cdot, \cdot \varepsilon$ & $\cdot, \ldots 1$ & الالالة المعنوية & \\
\hline
\end{tabular}

من الجدول السابق للعلاقة الارتباطية بين البيئة الاجتماعية وظهور اضطرابات نفسية يتبين الآتي: هن ك توجد علاقة إرتباطية ذات دلالة معنوية عند مستوى معنوية (0. . • ) بين البيئة الاجتماعية ومقياس إضطراب الاكتئاب لعينة (الأحياء الفقيرة) حيث بلغت قيمة الدلالة المعنوية

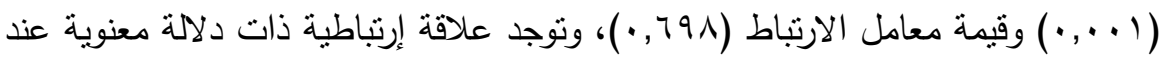
مستوى معنوية (0., •) بين البيئة الاجتماعية ومقياس إضطراب الاكتئاب لإجمالي العينة

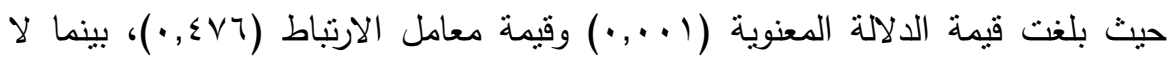
توجد علاقة إرتباطية ذات دلالة معنوية عند مستوى معنوية (0. • •) بين البيئة الاجتماعية

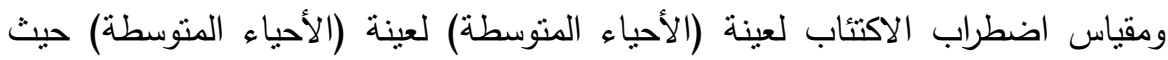
بلغت قيمة الدلالة المعنوية ( (, • ) وقيمة معامل الارتباط ( • 165 ( . ). 
ك توجد علاقة إرتباطية ذات دلالة معنوية عند مستوى معنوية (0. . •) بين البيئة الاجتماعية ومقياس إضطراب القلق لعينة (الأحياء الفقيرة) حيث بلغت قيمة الدلالة المعنوية (1 ( ., •)

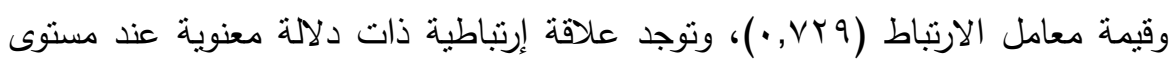
معنوية (0. . •) بين البيئة الاجتماعية ومقياس إضطراب القلق لعينة (الأحياء المتوسطة)

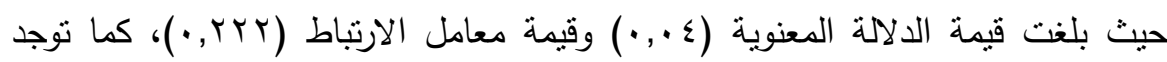
علاقة إرتباطية ذات دلالة معنوية عند مستوى معنوية (0., •) بين البيئة الاجتماعية

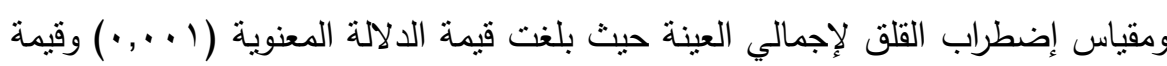

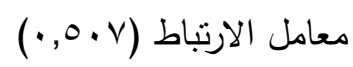

يمكن تفسير النتائج التي توصلت إليها الدراسة الحالية بانه يوجد علاقة بين متغيرات

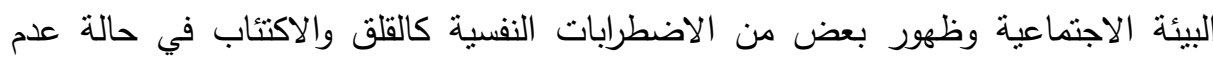
الرضا عن الحياة الاجتماعية التي يعيشها الفرد ويتضح دللك في الحياة الاجتماعية المحيطة بالفرد كالاسرة وعلاقته بالاخريين. وتتفق هده التتيجة الى بعض من الدارسات السابقة التي عرضتها الباحثة: دراسة ميشيل هيجرتي حول الرضا عن الحياة 999 19: حيث اثنارت تلك الدراسة الى ان هناك افراد يقومون بالحكم عن الرضا عن حياتهم من خلال خبراتهم الماضية وعلاقتهم بالافراد الاخرين ونصيب الفرد من الدخل القومي. دراسة كاتلين وكريستوفر ץ . Y. وأوضحت النتائج ان الأفراد الدين يندمجون في الأنشطة الاجتماعية بصورة اكبر ومقابلة الأصدقاء هم الدين يستمتعون بنوعية حياة أفضل.

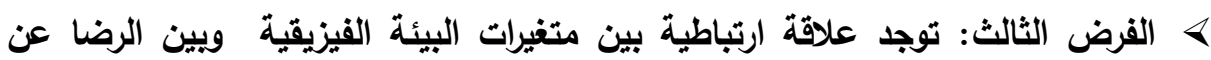
الحياة. 
أحمد مصطفى العنيق وآخرون

جدول(r): العلاقة الارتباطية بين الضغوط البيئة الفيزيقية للمسكن والرضا عن الحياة

\begin{tabular}{|c|c|c|c|c|}
\hline \multicolumn{3}{|c|}{ مقياس نوعية الحياة الفيزيقية للمنطقة السكنية } & & \multirow{2}{*}{ 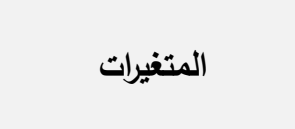 } \\
\hline إجمالي العينة & أحياء فقيرة & أحياء متوسطة & & \\
\hline ("*)., r). & $\cdot, 117$ & ("*)., sor & معامل الارتباط & \multirow{2}{*}{ الرضا عن النفس } \\
\hline$\cdot, \ldots 1$ & $\cdot, r$ & $\cdot, \ldots 1$ & الدلالة المعنوية & \\
\hline$\cdot, 170$ & $\cdot, \cdot 9 r$ & $(* *) \cdot$, (*q & معامل الارتباط & \multirow{2}{*}{ الرضا عن الآخرين } \\
\hline$\cdot, r$ & $\cdot, \varepsilon$ & $\cdot, \ldots 1$ & الدالة المعنوية & \\
\hline סטז, • ("*) & $\cdot, 1<9-$ & $(* *) \cdot, 7) 7$ & معامل الارتباط & \multirow{2}{*}{ تقبل مستوى المعيشة } \\
\hline$\cdot, \ldots 1$ & $\cdot, r$ & $\cdot, \ldots 1$ & الدلالة المعنوية & \\
\hline$\left({ }^{* * *} \cdot, \leq Y\right)$ & $\cdot, \cdot$. & $(* *) \cdot, 719$ & 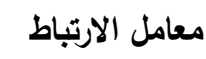 & \multirow{2}{*}{ قياس الرضا عن الحياة } \\
\hline$\cdot, \cdots$ & $\cdot, \mathrm{V}$ & $\cdot, \ldots$ & الدلالة المعنوية & \\
\hline
\end{tabular}

من الجدول السابق للعلاقة الارتباطية بين متغيرات البيئة الفيزيقية للمسكن وعدم

الرضا عن الحياة يتبين الآتي:

ك توجد علاقة إرتباطية ذات دلالة معنوية عند مستوى معنوية (0., ·) بين مقياس نوعية الحياة الفيزيقية للمسكن والرضا عن الآخرين لعينة (الأحياء المتوسطة) حيث بلغت فيمة

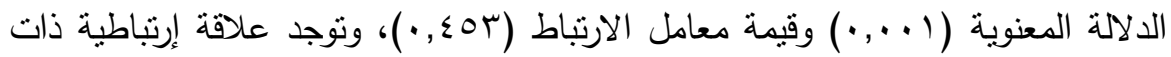

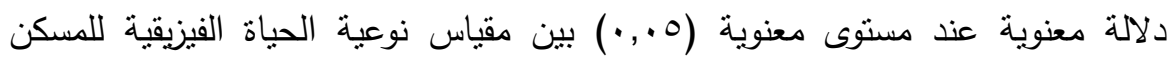
ومقياس إضطراب الاكتئاب لإجمالي العينة حيث بلغت قيمة الدلالة المعنوية (1 (.,.•) وقيمة معامل الارتباط (10, (·)، بينما لا توجد علاقة إرتباطية ذات دلالة معنوية عند

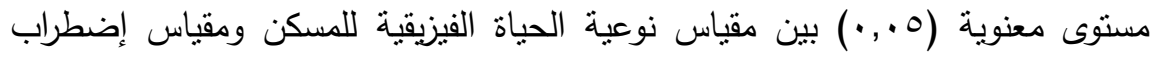
الاكتئاب لعينة (الأحياء الفقيرة) حيث بلغت قيمة الدلالة المعنوية (V +., ) وقيمة معامل

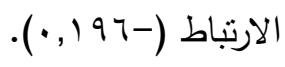

\& توجد علاقة إرتباطية ذات دلالة معنوية عند مستوى معنوية (0., •) بين مقياس نوعية

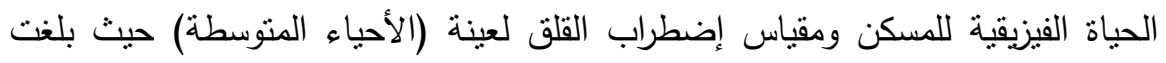

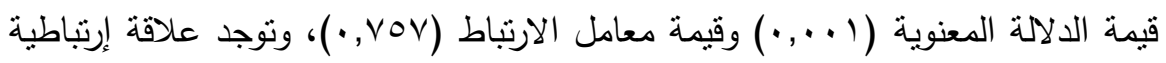


ذات دلالة معنوية عند مستوى معنوية (0., •) بين مقياس نوعية الحياة الفيزيقية للمسكن

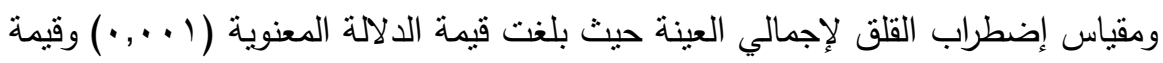

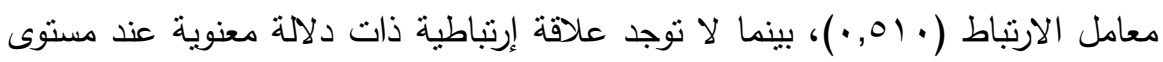

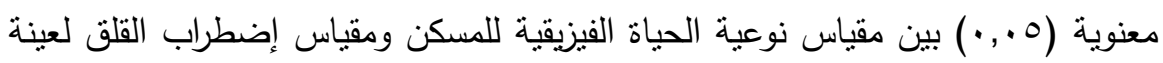
(الأحياء الفقبرة) حيث بلغت قيمة الدلالة المعنوية (^•,.•) وقيمة معامل الارتباط $\cdot(\cdot, 1 \wedge 9)$

أوضحت الدراسة الحالية ان مواصفات المسكن الدي يعيش فيه الفرد وشعوره بالامن

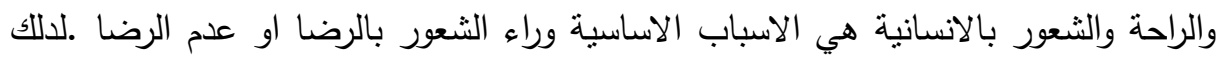

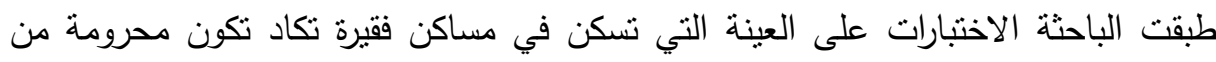
المسكن المناسب وهي العينة التي بيئتها الفيزيقية غير صحية لحياة مناسبة للتكيف السليم وتعاني من الازدحام وسوء حالة المسكن ونقص الخصوصية ونقص الخدمات والمرافق والعينة الاخرى هي التي كانت تسكن بالسكن المناسب للحياة الصحية السليمة وكان السكن في حي

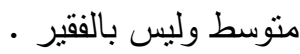
وقد أثنارت الباحثة من خلال عرضها للدراسات السابقة اهية البيئة الفيزيقية في تاثيرها على شعور الفرد بالرضا عن الحياة .

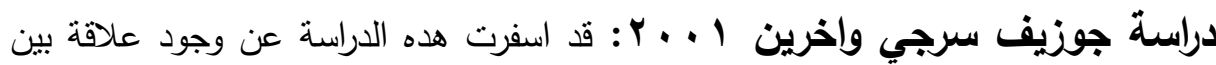
البيئة الفيزيقية التي يعيش فيها الفرد وبين تحقيق الرضا عن الحياة والصحة النفسية حيث اوضحت ان اثباع الحاجات المختلفة كالبيئة المحيطة بالفرد والجيران تتعكس على شين شعوره بالرضا عن الحياة. دراسة مارجريت بولما وجيمز ليكولو999 19 1ا والتي توصلت نتائجها الى ان التدين المفرط هو الافضل لنقبل الحياة والرضا عنها لفترات عمرية اطول. 


\section{اللزوسيان}

على وفق ما توصل اليه هدا البحث من نتائج فان الباحثة تضع التوصيات الاتية: 1-الاهنمام بتوفير المسكن المناسب للحياة الصحية السليمة لشعور الفرد بالحياة الانسانية الكريمة حيث يتوفر في المسكن الخدمات والمرافق اللازمة للابتعاد عن عشوائية السكن. r-ثوفير دخل مناسب للفرد ليصبح راضيا عن حياته والتقليل من احتمالية تعرضه للاضطربات النفسية.

r-التوصية باهتمام الحكومة بتوفير المسكن الملائم لهذه الاحياء وتوفير الحياة الملائمة لهم. ع- توجيه الاعلام لنسليط الضوء على مساكن الاحياء العشوائية المحرومة من الخدمات والمرافق. - مون. 0-زيادة النواجد الامنى بنلك المناطق العشوائية للحد من العنف والجريمة.

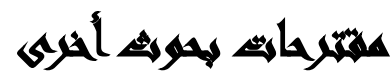

1 - إجراء دراسة مقارنة في الرضا عن الحياة بين النساء والرجال. ץ- إجراء دراسة في جودة الحياة والسلوك الاجتماعي بين بيئات ثقافية مختلفة في المجتمع.

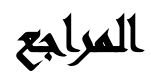

سعيد فرج(9V7 ( ) ): دراسات فى المجتمع المصرى، الهيئة المصرية للكتاب، الإسكندرية

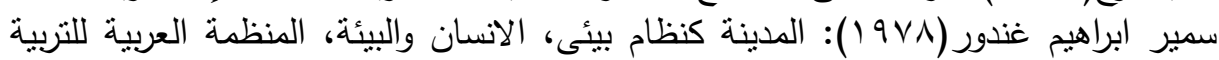

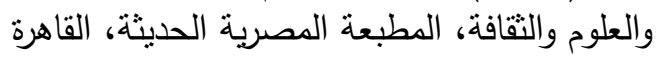

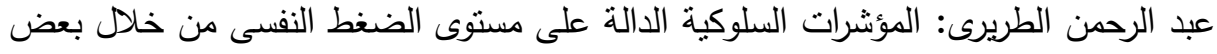

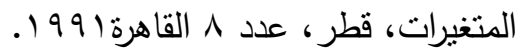

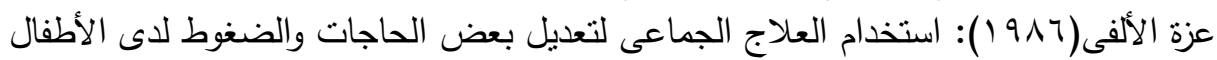

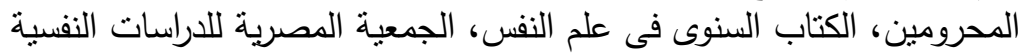

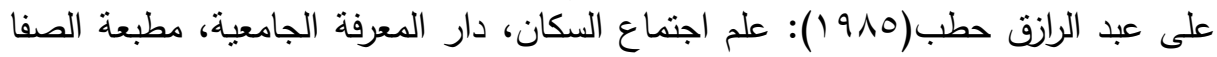
الحديثة، الأسكندرية (1) النية

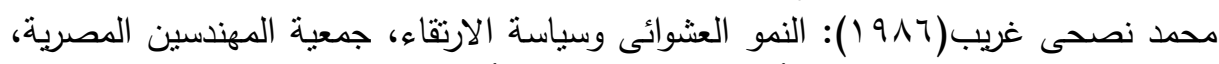
المؤتمر السنوى الأول لتخطيط المدن والأقاليم

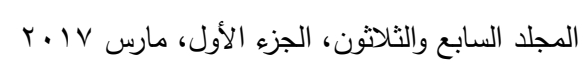


Ronald. J et al: "Sex Difference in Adolescent Life Stress Social Support and well being". J. of psychology, 98, 227-228, (1978).

Sarason I.G. et al.: Assessing the impact of life changes in I.G. sarason Cd. Spiel Berger (Eds) stress and Anxiety (Vol. 6) New York: Hemisphere Publishing Corporation, (1979).

Kessler, R.C: Stressful Life event Personality and Health. Journal of personality and social psychology, Vol.37, (1982).

Edward's: The Effects of Stress Management Curriculum of Elementary School children. In Desperation Abstract International (1987), vol. 48 (1), p. 43.

Lowrence, et al.: Life Stress and Adjustment Effects of the events experienced by young adolescents and their parents. Development. Psych, 23(4), 583, (1987). 
أحمد مصطفى العثيق وآخرون

\title{
THE RELATIONSHIP BETWEEN THE QUALITY OF LIFE AND THE EMERGENCE OF CERTAIN TYPES OF MENTAL DISTURBANCES AMONG THE POPULATION OF THE SLUMS IN THE CITY OF
}

\section{CAIRO}

El Atik, A. M. ${ }^{(1)}$; Hussein, M. A. ${ }^{(2)}$ and El Adawy, Somaya, I. S. 1) Institute of Environmental Studies and Research Research Ain Shams University 2) Faculty of Arts, Mansoura University

\begin{abstract}
There is no doubt that poverty as one of the environmental problems of the most important role to play roles in the reality of contemporary Egyptian life. And poverty converts between man and the achievement of economic and social level desirable and acceptable. Developing countries experiencing the phenomenon of inflation and growth of urban growing in a continuous and increasing population density and the approach of the population in many urban environments, to the point of saturation The result was the emergence of many environmental problems that afflict these environments, the most important problem of housing, transportation and non-availability of facilities and basic services in these cities There are areas in the city of Cairo is the environments in which the deprivation of basic needs of life and health, social and psychological comfort and low economic level, which are impeding the adjustment to achieve a psychological sense and appropriate behavior and the formation of positive attitudes towards society and towards the other These areas have several pictures are in the graves population, slum dwellers and the margins are here to point out the need to provide adequate housing for the upbringing of children as provided by the housing of the safety, comfort and

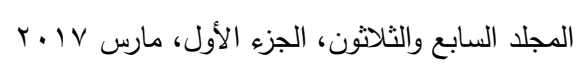


relaxation and a sense of humanity to enjoy mental health. These areas lack basic services to utilities such as water, sanitation and electricity. Has made various research in these areas that $35 \%$ of the buildings do not reach them with drinking water and that $55 \%$ of the buildings do not have waste water as well as deprivation of cleaning services.And lacking in these areas are also to recreational services, whether in the form of open recreational areas such as gardens, parks or sports fields. Or recreational areas such as the cinema closed.

So we find that there is no outlet for residents of the center of this massive accumulation of buildings and population in addition to the lack of reserves to face the problems that occur in the region like wildfire and the various incidents The quality of life in this slum areas is very low in many districts the standard of living for a human in terms of essential goods and basic services needed by the individual and which should not be this low level for a certain extent, a reduction which remains on the human life

The underdevelopment states witness currently the phenomenon of urban inflation, being continuously increasing in addition to the phenomenon of over population.

Research Objective: the current study drives at identifying one of the critical problems the Egyptian society is suffering from, namely, identifying the quality of life residents in poor quarters in Cairo (slums), and identifying the behavioral patterns of these areas' dwellers such as aspects of psychological disorders. Method: this research uses the descriptive (qualitative) method. Research Sample: the study sample consists of (180) families dwellers in Cairo city quarters, divided into (90) poor families and (90) middle-level families.

Research tools: the researcher uses several scales to achieve the study targets as follows:

1- Scale of Psychological disorders (depression - anxiety) - by researcher.

2- Questionnaire of quality of Life (analysis of the social environment and the physical environment) - by researcher.

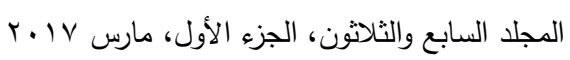


أحمد مصطفى العنيق وآخرون

The most important results: the study comes to these results:

1- There is a correlation between the environment's physical variables of the lodging and emergence of psychological disorders.

2- There is a correlation between the environment's social variables of the housing and emergence of psychological disorders.

3 - There is a correlation between the environment's physical variables and gratification of life.

\section{Recommendations:}

1- Consider providing the appropriate house for those people for a healthy life and feeling human, a house that should have services and utility.

2- Providing the appropriate income to make individuals satisfied for their lives and reduce the possibility of having psychological disorders. 